\title{
Spatial Summation of Optic Stimuli in the Human Retina as Revealed by Electrical Stimulation.
}

\author{
By
}

\section{Kōiti Motokawa.}

(本川弘一)

(From the Physiological Laboratory of Prof. K. Motokawa, Tohoku University, Sendai.)

(Received for publication, October 14, 1947)

There is much evidence which tends to show that spatial summation takes place in the human retina. For instance, there is an inverse relation between threshold intensity and size of area illuminated (Piper, ${ }^{11}$ Reeves, ${ }^{2)}$ Piéron $\left.{ }^{31}\right)$. Beitel ${ }^{4}$ presented two semi-circles separated by various distances and determined the intensity threshold as a function of the distance between the two stimuli. The result shows that when both semi-circles are presented together the threshold intensity increases as the stimuli are taken farther and farther apart up to the limit of separation of $2^{\circ} 30^{\prime}$, beyond which no interaction takes place any more.

As to the summation of over threshold stimuli, there are the experiments of Granit ${ }^{5)}$ and of Granit and Harper. ${ }^{6)}$ Granit determined flicker fusion frequencies for four circular test patches together and for each of the patches alone. With a higher intensity of illumination the fusion frequencies for all the patches were regularly 2.5 revolutions per sec. on an average higher than those of single patches at $10^{\circ}$ from the center. With central fixation there was much less summation under otherwise similar conditions. Granit and Harper found a linear semi-logarithmic relation between flicker fusion frequencies and area illuminated, and the slope of the curve to be a little steeper in the periphery than in the center. From these results Granit concluded that summation takes place in the periphery to a greater extent than in the center.

Motokawa ${ }^{78)}$ has shown that the effect of light can be measured quantitativêly by measuring increases in electrical excitability of the retina after illumination. Motokawa and Iwama ${ }^{9)}$ have demonstrated that the relation between the maximum increase of electrical excitability due to illumination and intensities of light is very similar to the relation between flicker fusion frequencies and intensities of illumination as studied by 
Hecht and Verrijp. ${ }^{10)}$ The present research has been undertaken to investigate spatial summation in the retina with this new method introduced by the author.

\section{MEthod.}

The details of the procedure involved are described in Motokawa's papers cited above so that they may be omitted in this report.

Large silver electrodes were placed at the outer corner of the left eye and over the left brow, the electrical contact between skin and electrodes being made with electrode paste of high $\mathrm{NaCl}$ concentration. A constant current pulse $100 \mathrm{msec}$. in duration was used as a test-stimulus, and the threshold was determined with light sensation due to electrical stimulation as the index. Measurements were performed in a darkened room after complete dark adaptation of the eye. The maximum of electrical excitability is usually reached in two seconds after the end of illumination when white light is used. So only the threshold at this moment was measured. arrangements for illumination will be described later.'

Let the electrical excitability or the reciprocal of threshold in the dark be $E_{0}$, and the excitability measured two seconds after the end of illumination be $\mathrm{E}$, then the excitability increase $\zeta$ is defined as follows:

$$
\zeta=\frac{E-E_{0}}{E_{0}} \times 100 \text {. }
$$

All the following results will be expressed in terms of $\zeta$.

\section{Results.}

1. Summation at Varying Distances between Stimuli.

Such an arrangement of test patches as indicated in Fig. 1 (A) was - used in this series of experiment. Each patch had a diameter of $1^{\circ}$ visual angle, and the distance $x$ between the central and peripheral circles was varied between $0^{\circ}$ and $6^{\circ}$. The central patch was always centrally fixated. The intensity of illumination was 1000 lux and the period of exposure 0.13 seconds.

The value of $\zeta$ was determined for the central patch alone and for all five together. The result is reproduced in Table $I$. The value of $\zeta$ for all the patches together is decidedly greater than that for the central patch alone at the fovea and parafoveal regions. Summation is weaker as the distance $x$ increases, and no indication of interaction is seen at a separation of $6^{\circ}$. So the limit of separation can be estimated at about $5^{\circ}$ for this intensity of illumination. In order to see how the limit of separation varies with intensities of illumination, similar measurements were made with intensities 5200, 280 and 180 lux at another subject, and the follow- 


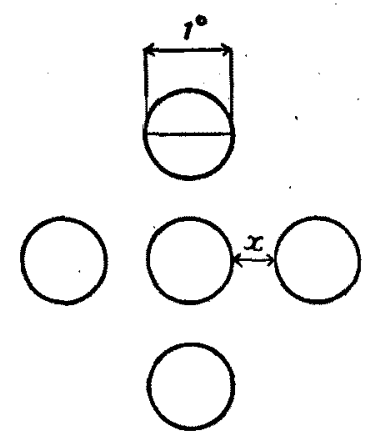

(A)

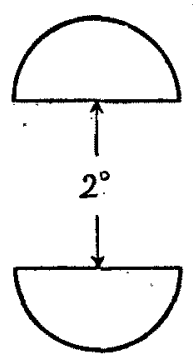

(B)

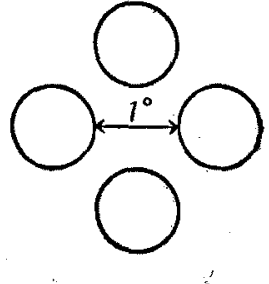

(c)

Fig. 1. Arrangements of test patches.

ing values were obtained: $13.6^{\circ}$ for $5200 \mathrm{lux}, 7^{\circ}$ for 820 lux and $3.5^{\circ}$ for 180 lux. Granit found fairly distinct summation in his "four-spot" flicker experiment with a separation of $2^{\circ}$ and an intensity of 100 lux. It seems,

\section{TABLE I.}

Summation effects at varying degrees of separation between stimuli

(five-spot experiment).

$x$ represents the nearest distance between the central and the other circles (arrangement of patches in Fig. 1 (A)). Intensity of illumination: 1000 lux. Exposure period: 0.13 seconds.

\begin{tabular}{c|c|c|c}
\hline$x$ in degrees & "Single" & "Five" & $\begin{array}{c}\text { Summation effect } \\
\text { (difference) }\end{array}$ \\
\hline 0 & 54.5 & 77.0 & 22.5 \\
1 & $"$ & 75.5 & 21.0 \\
2 & $"$ & 68.0 & 13.5 \\
3 & $"$ & 64.0 & 9.5 \\
4 & $"$ & 58.5 & 4.0 \\
6 & $"$ & 54.5 & 0
\end{tabular}

therefore, that the order of magnitude for the limit of separation agrees in both cases, though the experimental methods are quite different from one another.

\section{Summation at Various Parts of the Retina.}

Summation at various regions of the retina was examined with the arrangement of test patches shown in Fig. 1 (B). The result is shown in Table II. At the center, the value of $\zeta$ is 63.5 for the single semi-circle and 69 for both semi-circles together, the difference being 5.5. The values of $\zeta$ for singles and doubles decrease as the location moves farther and farther 
TABLE II.

Summation at various regions of the retina (semi-circle experiment). Distance between semi-circles: $2^{\circ}$ (Fig. 1 (B)). Illumination: 1000 lux.

\begin{tabular}{c|c|c|c}
\hline $\begin{array}{c}\text { Location } \\
\text { (degrees from } \\
\text { the center) }\end{array}$ & "Single" & " Double" & $\begin{array}{c}\text { Summation effect } \\
\text { (difference) }\end{array}$ \\
\hline 0 & 63.5 & 69.0 & 5.5 \\
20 & 50.0 & 55.5 & 5.5 \\
40 & 42.0 & 48.0 & 6.0
\end{tabular}

from the center, but the difference between double and single remains almost constant. If the difference is a faithful measure of summation the above result indicates that there is no local difference concerning summation. But the values of $\zeta$ decrease from the center towards the periphery so that the percentage increase of $\zeta$ by doubling the area must be greater for the periphery than for the center. The question as to which of these alternative interpretations is correct will be discussed in the following section.

This result has further been substantiated by another series of experiment which was performed with the arrangement of stimuli as illustrated in Fig. 1 (C). The result is reproduced in Table III. The values of $\zeta$ decrease as the measuring area moves from the center farther into the periphery, but the difference between the value for all the four patches together and that for the single varies only little like the result of above experiment.

\section{TABLE III.}

Summation at various regions of the retina (four-spot experiment). Arrangement of patches in Fig. 1 (C). Illumination: 1000 lux.

\begin{tabular}{c|c|c|c}
\hline $\begin{array}{c}\text { Location } \\
\text { (degrees from } \\
\text { the center) }\end{array}$ & "Single" & "Four" & $\begin{array}{c}\text { Summation effect } \\
\text { (difference) }\end{array}$ \\
\hline 0 & 41.0 & 68.5 & 27.5 \\
10 & 39.0 & 62.5 & 23.5 \\
20 & 35.0 & 58.5 & 23.5 \\
30 & 21.5 & 47.0 & 25.5 \\
40 & 17.5 & 39.0 & 21.5 \\
50 & 13.5 & 31.0 & 17.5
\end{tabular}

Illumination: 8 lux.

\begin{tabular}{r|r|r|r}
\hline 0 & 15.5 & 37.0 & 21.5 \\
10 & 14.0 & 23.5 & 9.5 \\
20 & 15.5 & 15.5 & 0 \\
30 & 8.0 & 6.0 & -2.0 \\
40 & 6.0 & 6.0 & 0
\end{tabular}


In the experiment with a much lower intensity of illumination some indication of summation is seen at the fovea and at $10^{\circ}$ from the center, but in the farther periphery there is no indication of summation at all. As shown in the report of Motokawa and Iwama, values of $\zeta$ for rods are generally much lower than those for cones; the former seldom exceed 30 . Therefore, it is difficult to show distinctly the summation for rod excitation in the periphere The fact that it has failed to prove any summation with the low intensity in the periphery, does not always mean that no summation takes place in the periphery, but only shows that the summation of peripheral cone excitation is weak or fails.

3. The Dependence of $\zeta$ on Area Illuminated.

The relation between $\zeta$ and area was quantitatively studied at the fovea and in the periphery. The size of patches was varied from $0.2^{\circ}$ to $3^{\circ}$ in diameter. The intensity of illumination was 1000 lux. The results are represented graphically in Fig. 2.

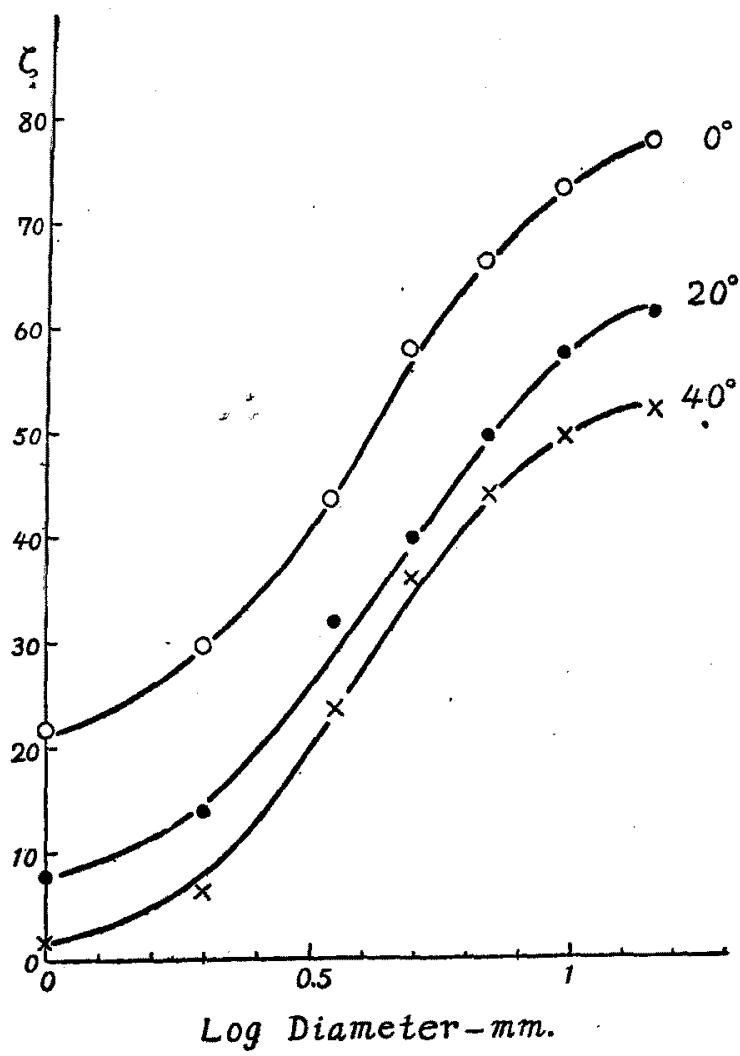

Fig. 2. Relation between the excitability increase $\zeta$ due to illumination and the logarithm of area illuminated. Intensity: 1000 lux. Period of exposure: 0.13 seconds. 
As seen from this figure, the shape of curves is always sigmoid just like the relation between $\zeta$ and $\log$ intensity as studied by Motokawa and Iwama. ${ }^{9}$ In the experiment of Granit and Harper, fusion frequencies of flicker were in a linear relation to the logarithm of area, and the slope of curves was steeper in the periphery than in the center. However, in my experiment the curves for the fovea and for the periphery $\left(20^{\circ}\right.$ and $40^{\circ}$ from the center) run almost parallel with one another, and in the midlle range of area in which a linear relationship approximately holds, the slope of the curves is almost the same for the fovea and the periphery. Thus, four experimental results seem to be inconsistent with those of Granit and Harper.

In the middle range of areas a geometric increase in area corresponds to an arithmetic increase in $\zeta$. So a definite amount of increase in $\zeta$ will be obtained by enlarging the area illuminated in a definite proportion, no matter what the initial value of $\zeta$ may be. This relation gives some clue to the question whether the absolute or relative (percentage) increase of $\zeta$ should be taken as a measure of summation. As stated in the preceding section, the percentage increase in $\zeta$ as the result of summation is greater in the periphery than in the center, but the absolute increase remains unaltered irrespective of the location. This relation is in entire harmony with the contsant slope of the $\zeta-\log$ area curves. All these results indicate that there are little regional differences, if any, with regard to summation, so long as our phenomenon is concerned. Further studies will be necessary to be able to answer the question whether the discrepancies between my results and those of Granit depend on the difference of methods or some other causes.

\section{Interaction between Areas Illuminated by Various Colored Lights.}

Motokawa $^{8 /}$ has shown that the time course of supernormal electrical excitability after illumination is characteristic of the wave-length of light. . It is of great interest to examine whether or not any interaction occurs between areas activated by lights of different colors. In this experiment two semi-circular patches of $2^{\circ}$ diameter in juxtaposition were used so as to confine the illumination to the rod-free part of the retina (Fig. 3). The approximate ranges of wave-lengths of the colored lights from the filters used were 620-740 $\mathrm{m} \mu$ for red, 490-550 $\mathrm{m} \mu$ for green and 410-490 $\mathrm{m} \mu$ for blue. So long as both semi-circles were illuminated by one and the same colored light the interaction between both areas was always marked for any sort of color, the value of $\zeta$ for both patches together being decidedly higher than that for any one patch viewed alone. For instance, $\zeta$ increased from 42 to 75 by doubling the area with red light, from 43 to 71 with green light and from 45 to 69 with blue light. On the contrary, there was no 
indication of summation at all when one semi-circle was illuminated by the red light and the other by the blue light.
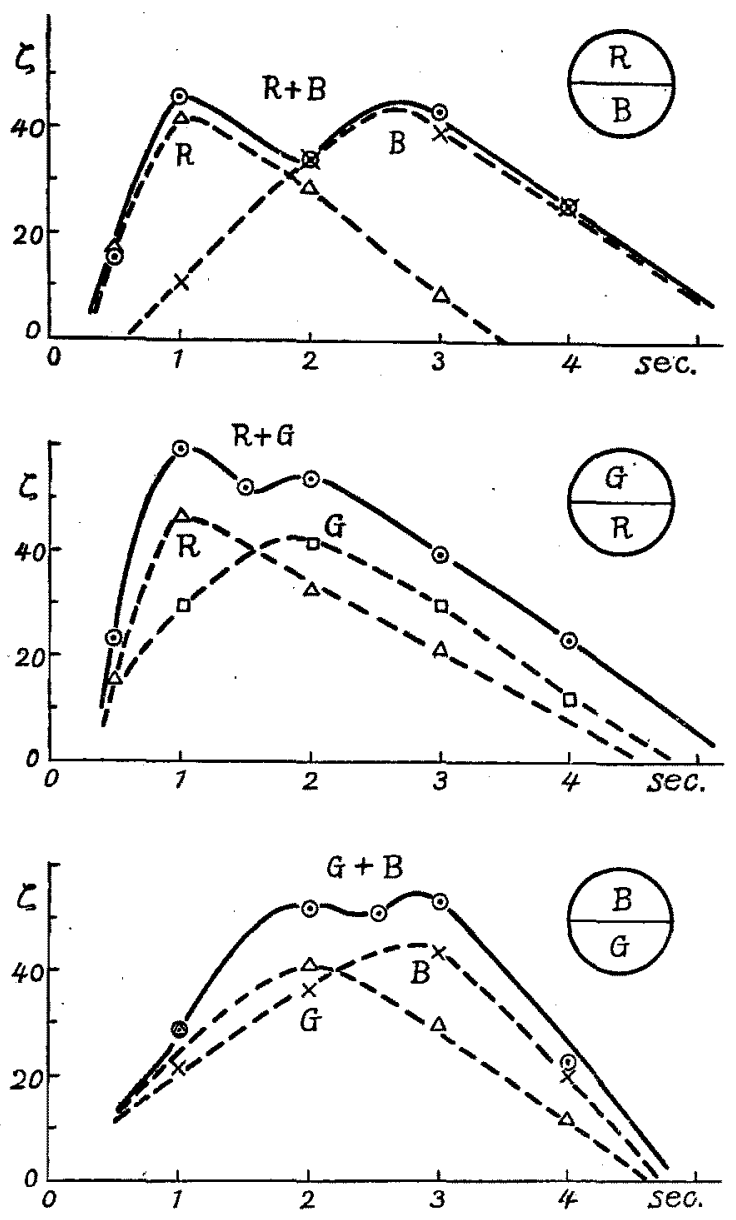

Fig. 3. Interaction between colored fields (semi-circles in juxtaposition). Abscissa: Time interval between optic and electric stimulation.

Ordinate: Percentage increase in electrical excitability after illumination.

Full lines: Summation curves. Broken lines: Curves for single patches.

R, G and $B$ mean red, green and blue lights respectively. The arrangement of the semi-circular patches is shown in the upper right corner of each figure.

The time course of the excitability curves for the "singles" and "doubles" is illustrated in Fig. 3 (the uppermost group of curves). As seen from this figure, the summation curve, $R+B$ which was obtained by simultaneous presentation of red $(R)$ and blue (B) semi-circles does not deviate greatly from the "single" curves, $R$ and $B$.

The middle group of curves in the same figure shows the interaction between areas excited by green and red lights. In this case the sum- 
mațion curve, $R+G$ is higher than any one of the "single" curves, indicating that there is some interaction between the red and the green semicircle. The increase in $\boldsymbol{\zeta}$ due to summation is about 13 and much smaller than the corresponding amount in the summation between areas of one and the same color, for instance 33 for red light and 28 for green light.

Between green and blue areas an interaction of the same order as in the previous case was proved (the bottom group of curves in Fig. 3). Generally speaking, the more akin the colors of both areas to each other, the more striking the interaction between them. The fact that there takes place little interaction between red and blue areas suggests that both sorts of light must involve different kinds of mechanisms which act independently of each other. Some degree of interaction proved between red and green or green and blue indicates that the pair of lights must call into play some mechanism common to each. After all, our findings fall into the scheme of one of the color theories such as those advanced by Young and Helmholtz or by Hering. They assume that there must be three or four kinds of mechanisms which act independently of one another.

If Young-Helmholtz's theory is adopted to interpret our findings, the scant interaction between red and blue areas may be explained as follows: The red light involves chiefly $\mathrm{R}$-component, but scarcely B-component, while the blue light stimulates B-component without involving R-component. As it is assumed in the theory that each component acts independently, there is no reason for interaction between the field in which only R-component is active, and the area in which only B-component is active. In reality, however, there is a slight interaction between red and blue area, what is evidenced by the fact that the summation curve, $R+B$ is slightly higher than the "single" curves. This may rest on the circumstances that red and blue lights can stimulate the component $G$ to a slight degree.

The interaction between red and green areas or green and blue areas may be ascribed to the properties of the green light to stimulate not only G-component but also $\mathrm{R}$ - and B-component to some extent.* In this way, we are led to the conception that summation or interaction occurs only among components or mechanisms of one and the same kind.

It is also worth while noticing that the theory of Young-Helmholtz applies to our findings which have been obtained without resort to any color sensation.

\section{Summary.}

Spatial summation in the human retina was studied with increases of electrical excitability by illumination as the index.

1. The maximum increase in electrical excitability is reached in 2. 
seconds after an illumination. This amount was expressed in percentage of the electrical excitability in the dark and denoted by $\zeta$. The value of $\zeta$ after an exposure to five circular patches $1^{\circ}$ in diameter was always higher than the value for a single patch of the same size. The closer the five patches to one another, the greater the value of $\zeta$.

2. Two semi-circles separated by an unstimulated region of $2^{\circ}$ was used to study summation at various parts of the retina. The value of $\zeta$ was greater by a constant amount for both semi-circles together than for a single semi-circle irrespective of the location in the retina.

3. $\zeta$-log area curves were sigmoid in shape and their slope was the same in the fovea and in the periphery. These findings suggest that there are little regional differences with regard to summation.

4. Two semi-circular patches in juxtaposition were employed to investigate the interaction between colored lights. Summation was most striking between same colored areas, and fairly marked between red and green fields or green and blue areas, but there was no indication of summation at all between red and blue areas. The result was explained on the basis of Young-Helmholtz's theory of color vision.

\section{References.}

(1) Piper, H., Zsch. f. Psychol., 1903, 32, 98.

(2) Reeves, P., Astrophys. J., 1918, 47, 141.

(3) Piéron, H., C. r. Soc. Biol., 1920, 83, 753.

(4) Beitel, R. J., J. Gen. Psychol., 1934, 10, 311.

(5) Granit, R., Am. J. Physiol, 1930, 94, 41.

(6) Granit, R. and P. Harper, Am. J. Physiol., 1930, 95, 211.

(7) Motokawa, K., Tohoku J. Exp. Med., 1949, 51, 145.

(8) Motokawa, K., Tohoku J. Exp. Med., 1949, 51, 165.

(9) Motokawa, K. and K. Iwama, Tohoku J. Exp. Med., 1949, 51, 155.

(10) Hecht, S. and C. D. Verrijp, J. Gen. Physiol., 1934, 17, 251.

* To interpret this phenomenon, physiological induction should further be taken into account, as has been reported by Motokawa and Iwama in a preliminary report (this journal, $1949,50,292$ ). 